alone or in combination with dexamethasone; however, the efficacy of lenalidomide in previously untreated multiple myeloma patients has not been studied. In a phase II clinical trial, Rajkumar and co-workers investigated toxicity and response rates in previously untreated, newly diagnosed multiple myeloma patients treated with lenalidomide plus dexamethasone.

In their study, patients $(n=34$, median age 64 years) were treated with a $25 \mathrm{mg}$ daily oral dose of lenalidomide on days 1-21 and a $40 \mathrm{mg}$ daily oral dose of dexamethasone on days 1-4, 9-12 and 17-20 of a 28-day cycle. Patients were treated with a minimum of four cycles. Objective responses to therapy, defined as a decrease of $\geq 90 \%$ or to $<200 \mathrm{mg} / 24 \mathrm{~h}$ in urine $\mathrm{M}$ protein, and a $\geq 50 \%$ decrease in serum monoclonal protein, were observed in $31 / 34$ patients (91\%). Of these responding patients, 2 had complete responses and 11 had very good partial or near complete responses. Side effects were manageable, with $47 \%$ experiencing grade III or higher nonhematologic toxicity, most commonly fatigue (15\%), muscle weakness $(6 \%)$, anxiety (6\%), pneumonitis $(6 \%)$, and rash $(6 \%)$.

The good response rates and relatively low toxicity observed with lenalidomide plus dexamethasone indicate that this regimen is a candidate for primary therapy of multiple myeloma; the efficacy of the treatment should be investigated in phase III trials.

Kate Matthews

Original article Rajkumar SV et al. (2005) Combination therapy with lenalidomide plus dexamethasone (Rev/Dex) for newly diagnosed myeloma. Blood 106: 4050-4053

\section{Intraperitoneal chemotherapy for ovarian cancer: challenges and future directions}

The high incidence of complications and toxicities associated with intraperitoneal chemotherapy (IPC) in women with ovarian cancer has previously been noted. Despite reports that IPC significantly improves progressionfree and overall survival when compared with systemic chemotherapy, many patients undergoing IPC do not complete therapy. In a recent Gynecologic Oncology paper, Walker et al. discuss patient characteristics and complications associated with the 205 women enrolled in the IPC arm of the phase III Gynecologic Oncology Group trial 172.

In this trial, following primary surgical tumor debulking, patients in the IPC arm received intravenous paclitaxel on day 1 , followed by intraperitoneal cisplatin on day 2 and intraperitoneal paclitaxel on day 8. Therapy was discontinued before completion of six cycles in $58 \%$ of patients; approximately half of the patients received four, five, or six cycles. Discontinuation of therapy was directly catheter-related in 34\% of patients, with complications including infection, and blocked or leaking catheters. The authors comment that surgeons will therefore need to improve their techniques for this therapy to be feasible. Among patients who had undergone a left colon or rectosigmoid colon resection, $16 \%$ of patients did not receive any IPC, compared with only $5 \%$ of those who had undergone an alternative surgical procedure $(P=0.01)$.

The authors conclude that delivery of six cycles of IPC is challenging in this patient population. Approximately one-third of the participants discontinued IPC because of cisplatin toxicity, and more attention to hydration, antiemetics and supportive care is needed.

Alexandra King

Original article Walker JL et al. (2006) Intraperitoneal catheter outcomes in a phase III trial of intravenous versus intraperitoneal chemotherapy in optimal stage III ovarian and primary peritoneal cancer: a Gynecologic Oncology Group study. Gynecol Oncol 100: 27-32

\section{Does concurrent exemestane affect tamoxifen pharmacokinetics?}

Adjuvant breast cancer treatments often act by reducing circulating estrogen levels or inhibiting estrogen receptor action. Preclinical studies have suggested that exemestane, which acts by suppressing estrogen synthesis, might have synergistic activity when combined with tamoxifen, a selective estrogen receptor modulator. Hutson et al. investigated the effect of exemestane on tamoxifen pharmacokinetics and tolerability.

This open-label study enrolled 32 postmenopausal women (median age 57 years) with stage I/II invasive breast cancer, who had 